\title{
La alimentación del lactante: la nodriza y el examen probatorio de la leche en la obra de Oribasio
}

\author{
The alimentation of the lactant: the nodrize and the milk \\ "probatory exam" in the Oribasio text.
}

\author{
Mercedes López Pérez \\ Universidad de Murcia
}

\begin{abstract}
RESUMEN
El presente trabajo tiene como objetivo principal analizar el examen probatorio de la leche de Mnesites de Cízico, que conocemos gracias a la obra de Oribasio, incluyo también la comparación con un capítulo que versa sobre el mismo tema en la obra de Sorano de Éfeso.

Estos textos están impregnados de «irracionalidades», que son mas propios de los conocimientos populares que de lo que entendemos como conocimiento científico. El cuerpo femenino continua siendo un gran desconocido en ésta época y sus cuidados están sobre todo relacionados con la salud doméstica.
\end{abstract}

ABSTRACT

PALABRAS CLAVE:

The present work has the principle objetives analysing «brest milk» of Menesites of Cízicus, that we know thanks to the works of Oribasius. Also, he includes the comparison between one chapter of the work of Sorano. These texts are complete with «irracionalities» that are know more clarly through the popular knowledge from de folk medicine.

\section{KEYWORDS:}

Oribasius of Pergamum. Mnesites of Cizicus. Soranus of

Ephesus. Wetnurses. Newborn children. Mother milk. Health. Medicine.

Oribasio de Pérgamo. Mnesites de Cícico.

Sorano de

Éfeso. Nodrizas. Recién nacidos. Leche materna. Salud. Medicina. 
El presente trabajo tiene como objetivo principal analizar los textos referentes al tema de la nodriza y la lactancia en el recién nacido recogidos en la obra de Oribasio". Estos textos pertenecen a los capítulos 13-16 de los «Libros Inciertos» de Oribasio en la edición de Bussemaker-Daremberg ${ }^{2}$, dichos capítulos también aparecen en la obra de Raeder ${ }^{3}$, considerándolos ambos editores como textos pseudoribasianos ${ }^{4}$.

Oribasio de Pérgamo (325-395/6 d.C), médico y amigo personal del emperador Juliano, escribió por encargo de éste la obra que lleva el nombre de Colecciones Médicas, también es autor de dos obras de reducido tamaño Sinopsis y Libros dedicados a Eunapio.

La Historia de la Medicina ha calificado a este autor de enciclopedista, quizá la relevancia de su trabajo no se deba a la prevalencia del principio de auctoritas en su obra, pero si debe ser considerado como una figura clave para entender la transmisión de la ciencia médica de época Antigua al mundo Medieval ${ }^{5}$. Oribasio junto con Aecio de Amida y Pablo de Egina pertenecen (en la perspectiva de la Historia de Ciencia) a la Antigüedad tardía, que gesta la Edad Media, época en la que se leen y se transmiten algunos textos clásicos hipocráticos y galénicos, entre otros, con otra perspectiva y bajo una mirada muy distinta de aquella para la que fueron escritos.

El estudio de la fisiología y patología femenina restringida al aparato reproductor de la mujer, el útero, así como los accidentes mas comunes relacionados con el embarazo, junto con la elección de la nodriza, la proliferación y benignidad de la leche son los temas femeninos por excelencia de los autores clásicos griegos y romanos ${ }^{6}$. La salud de la mujer, como ha dicho D. Gourevitch, es identificada a través de la salud de su útero, pero en el caso de las mujeres esclavas el órgano importante ya no es la matriz sino el seno ${ }^{7}$.

1 A. H. M. Jones et alii, The Prosopography of the Later Roman Empire, Cambridge University Press, 1975, vol. I, p. 15.

2 U. C. Bussemaker y Ch. V. Daremberg, Oribase. Oeuvres complètes avec texte grec et traduction française établie par les docteurs Daremberg et Bussemaker, I-VI, Paris 1851-1876.

${ }_{3}$ Oribasius: Collectiones medicae. Edición de J. Raeder, Oribasii collectionum medicarum reliquae, [Corpus medicorum Graecorum, vol. 1-5. Leipzig: Teubner, 1926-1933]. Existe reedición anastática de1964.

${ }^{4}$ No es objetivo de este trabajo entrar en detalles sobre los textos pseudoribasianos de ambos editores, para un comentario mas detallado a dichas ediciones se puede consultar el capítulo V L'Enseignement de puériculture de Mnésithée epígrafe A La sholie de l'extrait 37 des Livres Incertains d'Oribase, en J. Bertier, Mnésithe et Dieuchès, J. Brill, Leiden, 1972.

${ }_{5}^{5}$ M.H. Green, The Transmisión of Ancient Theories of femaly physiology and disease through the Early Middley Ages, Princeton University, 1985

6 Tratados Ginecológicos: Sobre las enfermedades de las mujeres, Sobre las mujeres estériles, Sobre las enfermedades de las vírgenes, Sobre la superfetación, Sobre la escisión del feto, Sobre la naturaleza de la mujer en Tratados Hipocráticos IV, Biblioteca Clásica Gredos, Madrid 1988, y Generación, Naturaleza del Niño, Enfermedades IV, Parto de ocho meses, Parto de siete meses en Tratados Hipocráticos VIII, Biblioteca Clásica Gredos, Madrid 2003.

7 D. Gourevitch, «L'alimentation du petit enfant Romain »en Revue International de Pediatrie 39, 1988, pp. 43-46. 
La inquietud por la selección de una buena nodriza y la alimentación del recién nacido durante los primeros meses son una constante en los textos referidos a la salud femenina. La calidad de la leche, los alimentos galactogogos, el miedo a perder el alimento, en una sociedad tradicional que no posee otras alternativas ${ }^{8}$, hace que dichas cuestiones sean planteadas y recogidas en los textos dedicados a la salud de la mujer. Estos temas pertenecen al ámbito de lo estrictamente femenino y aunque están incluidos y son tratados por los expertos en medicina, sin embargo, dichas prácticas y consejos están rodeadas de supersticiones y elementos irracionales que han pervivido algunos de ellos durante siglos ${ }^{9}$ y que son propios de la medicina popular que participa del mismo espíritu precientífico que ha caracterizado a la ginecología y la tocología desde los Tratados Hipocráticos ${ }^{10}$.

La sangre al igual que la leche son fluidos fundamentales en el cuerpo femenino, necesarios para el mantenimiento del equilibrio, que en su defecto o superabundancia ocasionan la enfermedad. Ambos están estrechamente relacionados, la sangre muy abundante en la mujer, debido a su vida sedentaria y ociosa, si ella pertenece a las clases altas, es contrarestada y evacuada gracias a las reglas, pero en algunas mujeres la menstruación no es suficiente, se produce un aumento de plétora dando lugar a la obesidad y la enfermedad. Tras el parto, la sangre se transforma y se convierte en leche que alimentará al recién nacido ${ }^{11}$. Por eso ambas sustancias están estrechamente relacionadas con la salud de la mujer en diferentes etapas de su vida.

En este trabajo pretendo analizar las diferentes pruebas a las que debe someterse la leche para comprobar su calidad. El test mas completo es el que aparece en la obra de Oribasio y que se debe a Mnesites de Cízico.

Estas pruebas no parecen haber tenido el mismo grado de relevancia en la literatura médica, como lo demuestra el excelente trabajo sobre el tema de Amal Aboul $^{12}$. Este investigador señala como Mnesites considera la prueba como un ver-

8 El recién nacido romano es alimentado normalmente por su madre o por la nodriza, pero existen en la obra de Sorano y Mustio alusiones a objetos que desempeñan las funciones de tetinas artificiales son los llamados ubupa, vasos de vidrio con forma de mamelón agujereado, que difieren de los gutti, pequeños vasos que vierten el liquido de su interior gota a gota. Para completar este tema, ver D. Gourevitch, «L'alimentation artificielle du petit enfant dans l'Antiquité Classique», en Les biberons du Docteur Dufour, Museo de Fecam n. ${ }^{\circ}$ 18, 1987, pp. 13-18.

9 M. López Pérez, «La cultura popular natalicia: el arte de amamantar en el Campo de Cartagena», Revista murciana de Antropología, 2004, pp. 219-226. Se trata de un trabajo de campo que recoge las costumbres relacionadas con la lactancia en el Campo de Cartagena y su analogía con el mundo clásico.

10 En la obra de J. Joly, Le Niveau de la Science Hipocratique. Les Belles Lettres, París 1966, un clásico de la medicina hipocrática, donde el autor afirma en pág. 63: «En ninguna parte mas fácilmente que en ginecología y en tocología, dominios que afectan a la vida primitiva de la humanidad, la antigua medicina popular no tenía la ocasión de entender su dominio de la ciencia. Se trata por tanto de una misma familia de espíritu precientífico».

11 Mul. I, 73. Para la explicación fisiológica de la venida de la leche ver Sobre la naturaleza del niño 21, y Sobre las glándulas 17.

12 A. Amal Aboul, «Testing women's milk», Sciences exactes et sciences apliquees à Alexandrie, 1988, pp. 207-215. 
dadero método, Sorano le asigna un papel inferior, Galeno silencia el asunto. Las razones de estas diferencias son justificadas por el diferente grado de experimentación de la ciencia griega desde Hipócrates. Así pues, pretendo analizar las características de esos experimentos en Mnesites, que si bien son indicadores de cierta racionalización científica también incluyen un lenguaje simbólico o metafórico que camina a la par y que caracteriza a la ciencia griega desde sus inicios.

\section{LA NODRIZA EN LA DOCUMENTACIÓN LITERARIA}

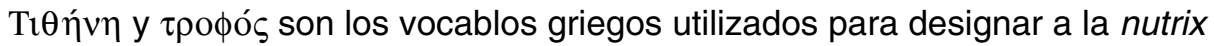
romana ${ }^{13}$. El término $\tau i \tau \theta \eta$, o ama de leche propiamente dicha se diferencia de

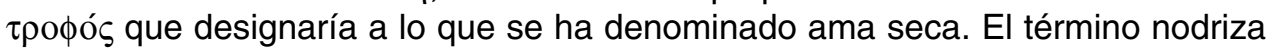
tendría dos acepciones: una amamantar a hijos ajenos y la otra ayudar en la crianza del recién nacido. Esta dicotomía en el lenguaje aparece ya en los poemas

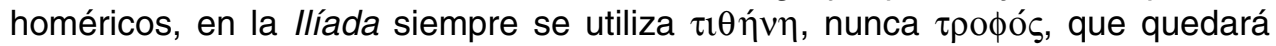
luego reservada a las nodrizas de los dioses ${ }^{14}$. La nodriza de época homérica es una nodriza ilustre, como lo indica la importancia que el aedo da a la nobleza de su linaje, resaltando para ello el nombre propio. Euriclea, es la nodriza de Ulises, ella es el prototipo de nodriza, y la esclava Eurimedusa la que alimentó a Nausicaa. También sabemos que otros héroes fueron amamantados por sus propias madres, tal es el caso de Hécuba que alimentó a Héctor y Penélope a su hijo Telémaco.

No es el objetivo de este trabajo el estudio de la nodriza en la literatura, pero es necesario resaltar que la literatura griega desde Homero hasta la poesía helenística se encuentra plagada de alusiones y referencias a estas mujeres que alimentan y crían a hijos ajenos. La nodriza que aparece en las fuentes literarias no es solo la que amamanta sino que en muchos casos acompaña y forma parte de la familia, le unen lazos afectivos con su «hijo» hasta el final de sus días.

La literatura griega recoge una variedad de estereotipos de nodrizas, desde esa imagen idílica de Euriclea hasta las nodrizas un tanto distorsionadas, pero en última instancia retratos de mujeres perezosas, indolentes y aficionadas al vino como aparece en algunos escritos de la comedia nueva representados por la obra de Menandro ${ }^{15}$. Algunas de estas características peyorativas aparecerán criticadas en los textos médicos, no directamente, pero si en esas recomendaciones morales que se le exigen a la nodriza.

13 Sobre las variantes, ver O. Navarre, «Nutrix», en Daremberg, Ch. y Saglio E. (eds.), Dictionnaire des Antiquités Grecques et Romaines, IV.1, Paris p. 122-123.

14 T. Molinos Tejada, «Madres y nodrizas en la Antigüedad», en: A. Pedregal Rodríguez y M. González González (eds.): Venus sin espejo. Imágenes de mujeres en la Antigüedad clásica y el cristianismo primitivo, Oviedo 2005, pp. 57-79 [p.63]. Para un estudio de la evolución de la madre y la nodriza en la literatura griega desde Homero hasta la poesía helenística consultar el excelente trabajo de T. Molinos Tejada, «Madres y nodrizas en la Antigüedad», en A. Pedregal Rodríguez y M. Gónzalez Gónzalez, (eds.), Venus sin espejo, pp. 57-79. Ver también M. García Sánchez, Las mujeres de Homero, Valencia 1999, p. 111 (citas de homéricas a las nodrizas).

15 Molinos, 2005, p.74. 
Es una idea frecuente relacionar la práctica de utilización de la nodriza en las familias de clases altas, pero ésta es una idea que debe revisarse, ya que en todas las sociedades antiguas y en todas las clases sociales la utilización de la nodriza ha sido necesaria como consecuencia de las defunciones de madres en el parto, la carencia de leche de la propia madre y la falta de otras alternativas, o por otras causas como ha indicado ${ }^{16}$.

La utilización de la nodriza parece ser habitual y progresiva desde finales de la Republica y durante el Imperio como queda manifestado por alusiones en las obras de Plutarco, Cicerón, Lucrecio ${ }^{17}$. Además hay que añadir el abundante material epigráfico de época romana, documentación que pone de manifiesto, sin duda, la proliferación y la utilización de una mujer ajena para la alimentación del recién nacido. Como lo demuestran las fuentes, parece ser más frecuente en el mundo romano que en el ámbito cultural griego ${ }^{18}$.

\section{LA LACTANCIA Y LA ELECCIÓN DE LA NODRIZA EN LOS TEXTOS OBSTÉTRICO-GINECOLÓGICOS}

La alimentación o el régimen, la diaita, es una noción antigua que incluye el régimen de vida, abarcando no solo lo relativo a los alimentos sino también a los ejercicios, los baños, etc. ${ }^{19}$. La alimentación se convierte en una preocupación porque es un aspecto fundamental para el equilibrio y la armonía, principios en los que se fundamenta el concepto de salud griega.

La alimentación del recién nacido aparece siempre en relación con los temas de salud femenina, interés que queda plasmado en los textos de Sorano de Éfeso y Oribasio, por resaltar tan solo algunos autores que manifiestan una relevancia especial para el tema que nos ocupa ${ }^{20}$.

16 J. Mangas Manjarrés, «Promoción social y oficio de las nodrizas», en Las edades de la dependencia durante la Antigüedad, Madrid, 2000, pp.223-238.

17 Mangas Manjares, 2000, p.223.

$18 \mathrm{~S}$. Crespo Ortiz de Zárate, Nutrices en el Imperio romano I. Estudio de las fuentes y prosopografía. Valladolid, 2005, p.11. Este exhaustivo trabajo que recoge la traducción de todo el material epigráfico latino sobre las nutrices romanas.

19 A.-L. Rey, "Autour des nourrissons byzantins et de leur régime», en V. Dasen (ed.), Naissance et petite enfance dans L'Antiquité. Collection Orbis Biblicus et Orientalis, 2003, pp. 363-375 [p. 363]. En este artículo se da una visión a la lactancia una dimensión ideológica analizada a través de textos patrísticos y hagiográficos.

${ }_{20}$ Existen trabajos realizados sobre la nodriza y las principales enfermedades infantiles en la obra de Galeno, para ello consultar D. Gourevitch, «Le nourrisson et sa nourrice: étude de quelques cas pédiatriques chez Galien», Revue de philosophie ancienne 19(2), 2001, pp.63-76. 


\section{La lactancia en Mnesites de Cízico}

Mnesites de Cízico ${ }^{21}$ es un médico que junto con Hipócrates, Diocles, Praxá-

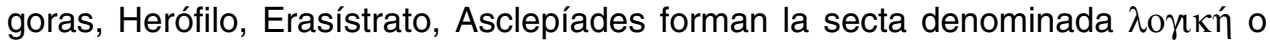

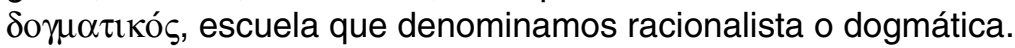

La obra de Mnesites queda incluida en esa gran laguna de documentación que afecta sobre todo al pensamiento científico entre Hipócrates y Galeno. Su cronología presenta algunas dificultades, aunque casi con toda probabilidad pertenece al primer tercio del siglo IV, como ha demostrado J. Bertier ${ }^{22}$. Su obra la conocemos por los fragmentos citados en Pausanias, Galeno, Ateneo, Sorano, Plinio y Oribasio, entre otros.

En el presente trabajo analizaré el capítulo 15 recogido en las Colecciones Médicas y catalogado como «libro incierto» por Daremberg y Bussmaker (1851-1876, vol. III). Como Anexo I aparece al final de este trabajo mi traducción de los fragmentos que he considerado más importantes para el análisis de dicho capítulo. La relevancia de estos fragmentos viene determinada por el contenido de los mismos en relación al tema que nos ocupa, la nodriza y la alimentación del recién nacido, pero concretando aún más, aquellos textos que hacen referencia a las pruebas que determinan la calidad de la leche.

Mnesites comienza su capítulo recomendando como nodriza a una mujer tracia o egipcia, la preferencia de estas mujeres no está justificada, aunque aparecen en la obra de Oribasio alusiones a la fertilidad de las mujeres egipcias, por la cercanía del fértil río Nilo. Sorano de Éfeso recomienda como nodriza una griega que conozca la más bella de las lenguas. La nodriza será una mujer sana, exenta de enfermedades cuya patología sea ha relacionado con creencias irracionales o de origen divino: la epilepsia y la sofocaciones uterinas.

El carácter y la personalidad de la nodriza son rasgos comunes en Mnesites y Sorano, era una creencia muy común el pensar que la nodriza transmitía su carácter a través de la leche. En la República, Platón ${ }^{23}$ señala la dificultad que con lleva la elección de una nodriza y en las Leyes $^{24}$ se recogen sus funciones. Estas exigencias comunes que insisten en la personalidad de la nodriza pueden deberse a que existe una estrecha relación entre la educación y la higiene ${ }^{25}$.

El verdadero interés del texto de Mnesites que conocemos por Oribasio radica, sin duda, en esas pruebas que el autor considera fundamentales para determinar la calidad de la leche, pruebas concretas, que no aparecen tan detalladas en ningún otro texto. Además de la observación por los sentidos, nuestro autor describe

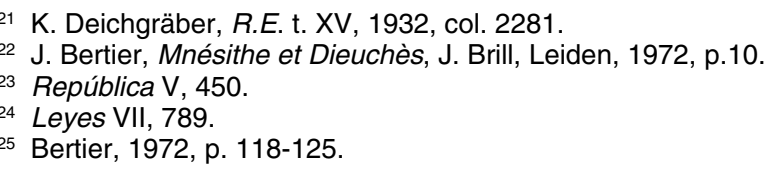


cuatro pruebas a realizar: la primera, debe realizarse para comprobar la dispersión de la leche en agua, la segunda es para comprobar la transparencia y el espesor, la tercera comprueba los elementos acuosos o gaseosos de la leche, y la cuarta debe realizarse para ver las características del coagulo de leche.

Estos experimentos son sin duda una paso hacia delante en la ciencia griega, pero es significativo comprobar como esa «racionalidad» está rodeada de exigencias que escapan a cualquier lógica. Dicho de otro modo, existen en la descripción de estas pruebas, una serie de rasgos que nos hacen pensar en esos principios que rigen la medicina natural: simpatía, antipatía, contigüidad, similaridad, y contrariedad $^{26}$.

Mnesites exige que el vaso o cuerno en el que se realicen las pruebas debe ser blanco, las gotas para los experimentos deben ser 10 o 15 y la octava parte de una cotila, uno de los experimentos debe realizarse en la uña del dedo pulgar, la cuarta prueba debe realizarse en primavera y llenar el vaso por la noche. $Y$ en relación a las exigencias generales a la nodriza: el último niño de ésta deberá ser de la misma edad y sexo que el de la madre, se debe amamantar tras los cuarenta días.

Estas ideas ponen de manifiesto, como ha dicho Bertier ${ }^{27}$, que la represión de lo irracional no significa la victoria de la racionalidad.

\section{Sorano de Éfeso}

El texto de Sorano de Éfeso es sin lugar a dudas el más conocido y estudiado como queda reflejado en la proliferación y relevancia de trabajos en los últimos años sobre su obra ${ }^{28}$. Sorano de Éfeso es médico y obstetra del siglo II preocupado por la salud de las mujeres escribe ${ }^{29}$ Las enfermedades de las mujeres. Una obra enteramente dedicada a la fisiología, patología y terapéutica femenina bajo un prisma metódico y en la que se eliminan cualquier concepción irracional o supersticiosa tan común en otros textos relativos a las mujeres. La obra de Sorano está dirigida a un público femenino, obstetras y parteras que deben ser bien entrenadas y educadas para conocer las alteraciones que son percibidas a través de los sentidos. Los deseos de Sorano aparecen en el primer capítulo de su primer libro, en donde se especifican las cualidades intelectuales, morales y físicas necesarias

${ }^{26}$ F. Gaide, «Aspects divers des principes de sympathie et d'antiphatie dans les textes thérapeutiques latins», en N. Palmieri (ed.), Rationnel et irrationnel dans la médecine ancienne et médievale. Aspects historiques, scientifiques et culturels. Université de Saint-Etienne, 2003, pp.129-144 [p. 137].

27 Bertier, 1972, p. XI.

28 La relevancia de estos estudios queda mas que demostrada en publicaciones como la de A.E. Hanson y M.H. Green, «Soranus of Ephesus: Methodicorum princeps», Aufstieg und Niedergang der Römischen Welt, II, 37.2, 1994, pp. 968-1037.

${ }_{29}$ P. Burguière y D. Gourevitch, Soranos d'Éphèse. Maladies des femmes, I-IV. Paris, Les Belles Lettres, 1990. 
para ejercer una profesión en un campo mucho más amplio que el de nuestros días. No debemos olvidar como ya señaló Lloyd ${ }^{30}$ curar la enfermedad en Grecia era competencia de al menos cinco grupos: los rizotomoi (cortadores de raíces), pharmakopai (los vendedores de drogas), los vendedores itinerantes de purificaciones, aquellos que curaban en los templos. las mujeres curanderas (maial) que se ocupaban de otras enfermedades femeninas además de asistir a los partos.

Las destinatarias principales de Sorano son las mujeres, encargadas de cuidar a otras y asistirlas en los partos en los que se producían una alta tasa de mortalidad infantil y femenina ${ }^{31}$.

Sorano de Éfeso aconseja a la mujer el retraso de su maternidad, espaciar sus embarazos y completar su educación. Se ha dicho de él que es el espíritu más práctico, más humano y utilizando un anacronismo, el más feminista ${ }^{32}$.

La obstetra ideal $(\tau \varepsilon \dot{\varepsilon} / \lambda \varepsilon i \alpha)^{33}$ debe ayudar a traer al mundo niños en las mejores condiciones de seguridad, debe ser una experta en enfermedades de la mujeres y practicar la obstetricia ${ }^{34}$.

Las exigencias a las nodriza están presentes en cuatro capítulos dedicados a la lactancia: la elección de la nodriza, el examen probatorio de la leche, el régimen alimentario, y como corregir las alteraciones de la leche ${ }^{35}$. Las prescripciones recomendadas a la nodriza incluyen: el habla griega, la edad, la experiencia, el físico, el estado de sus senos, el carácter, etc.

El capítulo nueve es el dedicado al examen probatorio de la leche, esta es una prueba necesaria para comprobar el buen estado de la misma. En este capítulo Sorano resalta tres formas de comprobar el buen estado de la leche: el primero, la nodriza debe tener las características anteriormente descritas, el segundo, si el niño está sano, esa es una buena señal, y la tercera prueba, la que hace referencia a la observación de la leche a través de los sentidos. Esta prueba consiste en examinar: el color, el olor, la consistencia, espesor, el sabor y la resistencia al tiempo de la leche. Este test, es tomado con toda seguridad de Mnesites, son co-

30 G.E.R. Lloyd, Las mentalidades y su desenmascaramiento, Madrid 1996, pp. 40-41. El mismo autor ha escrito un interesante trabajo dedicado al sexo femenino y su tratamiento: «The female sex: medical treatment and biological theories in the fifth and fouth centurias B.C.», en G.E.R. Lloyd, Science, Folklore and ideology. Studies in the Life Sciences in Ancient Greece, Cambridge University Press 1983.

31 B.D. Shaw, «The seasonal birthing cycle of Roman women», en W. Scheidel (ed.), Debating Roman Demography, Leiden 2001, pp. 83-109.

32 Burguière, 1988, p. XXXVI.

33 D. Gourevitch, «Un thérapeute accompli: note sur l'adjetif téleios» Revue de philologie 61, 1987, pp. 95-99.

${ }^{34}$ D. Gourevitch, «Préparation intellectuelle et déontologie de la sage-femme :du traité Des Maladies des femmes de Soranos d'Éphèse aux Infortunes de Dinah», en S. Kottek y M. Hortsmanshoff (eds.), From Athens to Jerusalem. Medicine in Hellenized Jewish Lore and in Early Christian Literature, 2000, pp. 69-73.

Maladies des femmes II, 8-11. 
munes en ambos autores la primera prueba, es decir, aquellos cualidades que deben ser observadas. En Sorano la observación de esas cualidades pasa a un segundo plano, las recomendaciones para la elección de la nodriza y el buen estado del niño ${ }^{36}$ son más importantes.

El color, el olor y la consistencia, son cualidades descritas en breves líneas, Sorano solo recurre a una prueba para comprobar la viscosidad y el espesor. Para reconocer el espesor se debe depositar una gota de leche sobre la uña o sobre una hoja de laurel o sobre una superficie lisa, se aplasta un poco y se conserva un poco para observar como se derrama. El resto de test que conocía por Mnesites no aparece en la obra, Sorano por tanto no da tanta importancia a las cualidades de la leche, para él las características de la nodriza y el buen estado del recién nacido son fundamentales.

Esta ambivalente actitud hacia el examen, como ha señalado Amal Aboul ${ }^{37}$, es un paso hacia delante una búsqueda para la específica cualidad de la leche, pero su falta de interés hacia el examen de la leche es una dificultad, que se justifica por la afinidad de Sorano hacia el Metodismo y la critica hacia algunos enunciados de los escritores del Corpus Hipocrático. Creo que esta hipótesis es del todo acertada y que se puede entender mucho mejor si señalamos como la excesiva importancia de la calidad y la cuantificación «aberrante» ${ }^{38}$ son una característica de los experimentos Hipocráticos, experimentos que son propios de una mentalidad precientífica, definidos en su mayoría por un sistema de cualidades basados en la experiencia común y en la intuición cotidiana. Y, sin embargo «la obra de Sorano está edificada según una lógica que es exactamente a la nuestra» ${ }^{39}$.

Volvamos al texto para comprobar con más facilidad estas ideas. Sorano al escribir sobre la calidad de la leche y sus pruebas, aunque conoce sin duda la literatura científica anterior, los Tratados hipocráticos y las pruebas de Mnesites, no puede argumentar a favor de ellas. Señala como Mnesites cuales son esas cualidades de la leche, a saber, el color, olor, consistencia, espesor, gusto y resistencia al tiempo, pero no se deja llevar por los experimentos que verifican esas propiedades. Es decir, la insistencia en el texto de Mnesites de utilizar un vaso de plata o cobre blanco ${ }^{40}$, que esos experimentos se hagan en primavera y por la noche. Sorano ${ }^{41}$ critica abiertamente a Mnesites por recomendar el vómito dos veces al día si se comprueba que la leche es demasiado fuerte, las razones de Sorano son de una lógica aplastante, el cuerpo se debilita. También son criticadas esas prescripciones que recomiendan la absorción de mamellas de animales grandes produc-

\footnotetext{
36 Maladies des femmes II, 9.

37 Amal Aboul, 1998, p. 213.

38 Joly, 1966, p.108.

39 D. Gourevitch, «L'alimentation du petit enfant Romain», Revue International de Pediatrie 39, 1988, p. LXXII.

40 Sin duda una analogía ente el color del vaso y la leche. Sustancia con la que se experimenta.

41 Maladies des femmes, II, 11.
} 
tores de leche ${ }^{42}$, así como ingerir lechuzas, murciélagos o espolvorear ceniza, la utilización de plantas aromáticas o píldoras de leche ${ }^{43}$ también es criticada.

Sin embargo a pesar de que la lógica y el espíritu de Sorano dista mucho del pensamiento de Mnesites, Sorano sigue compartiendo algunos principios de sus antecesores: la nodriza debe ser griega, debe estar en buenas condiciones psíquicas, no debe ser supersticiosa ni excesivamente religiosa, debe amamantar a un niño del mismo sexo que el suyo, de lo contrario los varones se afeminan y las hembras se masculinizan.

La nodriza y la alimentación del recién nacido forman como lo demuestra la documentación escrita y epigráfica, una constante en el pensamiento científico clásico. La pertenencia de dichos temas al ámbito de los cuidados femeninos, hace sin duda que muchos de estos principios, en ocasiones irracionales, sean propios de una especie de salud domestica femenina. Es significativo comprobar como la patología y sobre todo la terapéutica femenina está regida por principios más cercanos a la magia que a lo estrictamente científico. El cuerpo de la mujer en el mundo clásico es el gran desconocido, probablemente porque en la mayoría de los casos, las mujeres son cuidadas y tratadas por otras mujeres.

\section{APÉNDICE I.}

\section{De la leche de la nodriza y de la forma de reconocer la mejor leche. Tomado de Mnesites de Cízico}

\section{ORIBAS: Colecciones Médicas. Libros inciertos 15,1-7:}

Para alimentar a un niño, es necesario elegir una mujer de nación tracia o egip$\mathrm{cia}^{44}$, o cualquier otra que se le parezca. Ella deberá tener una gran talla, el pecho desarrollado, las carnes de buena naturaleza, ser bella, tomar todos los alimentos que le convengan y no padecer ninguna molestia en el vientre.

Estará exenta de cualquier enfermedad, y, sobre todo de epilepsia y sofocaciones uterinas, o de aquellas que se desarrollan por influencia divina.

Deberá vestir de forma adecuada así como cuidar otros detalles de su vida; su piel no tendrá mal olor; deberá tener un carácter alegre, fácil, dulce y simple; su edad no sobrepasará los 30 años y se quedará ella misma 1 ó 2 años; sus reglas no deberán aparecer durante la lactancia.

42 « II s'agit là d'une prescription magique typique, qui consiste à s'integrer en la mangeant la chose ou la qualité désirée« Maladies des femmes, II, p.103.

43 «Se conocen en Francia, hoy, bajo el nombre de Galactogil: una mezcla de Galega officinalis $L$., extracto de malta, esencia de hinojo, esencia de comino, vainilla y fosfato de cal». Tomado de Maladies des femmes II, p.102.

${ }^{44}$ La mujer egipcia era considerada una mujer muy fértil, cualidad que se le asignaba y compartía con las fértiles aguas del río Nilo. 
Ella será severa en sus relaciones con los hombres; ella habrá ya alimentado a varios niños, y su último niño deberá ser de la misma edad y del mismo sexo que el de la madre. Debe dar la leche tras cuarenta días después del parto pues es esta la mejor condición.

Preferimos sobre todo las mismas madres, si ésta no puede, las mujeres próximas, sus parientes, o las mujeres que se le parezcan por la forma.

\section{ORIBAS: Colecciones Médicas. Libros inciertos 15, 9-20:}

La mejor leche es aquella que ofrece las condiciones intermedias, en relación al espesor, cantidad, olor, color, gusto y espuma: si la cantidad no es mediana, se preferirá una nodriza que tenga mas leche.

La mejor prueba que se puede hacer es aquella que se hace con la ayuda de los sentidos; la segunda consiste primero en echar agua hervida, filtrada o lo mas pura posible, en un vaso de plata o de cobre blanco, provisto de una cavidad bastante grande para recibir el líquido en cantidad, después hacer caer en esta agua diez o quince gotas de leche; entonces se examinará si la dispersión de la leche en el agua no se produce ni extremadamente rápida, ni muy lentamente, sino con una celeridad media, esta es la mejor leche; es necesario hacer esta prueba en estado de salud.

Es aún una buena cualidad de la leche la de ser transparente sobre la uña: se deja caer unas gotas de leche sobre la uña del pulgar, y se la mirará el señalado, prestando atención al mismo tiempo a la forma en la que se desplaza, rápidamente, o lentamente, cuando baja hacia la uña, pues estos dos casos son igualmente malos; si ocurre al contrario, la leche se desplaza con una lentitud mediana es buena.

Se hará aun la prueba siguiente: se verterá la octava parte de una cotila de leche en un vaso de cristal o en un cuerno, o en una concha marina, y se añadirá una cantidad mediana, se aplastará con los dedos, después se dejará la mezcla hasta que se coagule; a continuación se examinará si se obtiene una cantidad mas abundante de elementos acuosos que de elementos gaseosos, pues una leche así no tiene nada de alimentos y la mayor parte pasa a la orina.

Si la leche cuajada contiene mas partes acuosas, es necesario creer que se digerirá y se elaborará difícilmente; la mejor leche es aquella que contiene una cantidad media de elementos gaseosos y una cantidad igualmente media de electos acuosos. Todavía es necesario hacer la prueba siguiente, sobre todo en primavera: por la noche se llena de leche el vaso de cristal o el cuerno, o la concha marina, se la coloca en un lugar bien expuesto al sol; por la mañana se examinará el coagulo, o la caspa, si es muy abundante, o si, al contrario, existe una pequeña cantidad, mientras que el líquido es abundante: estas dos clases de leche son malas; aquella, al contrario que presenta una buena proporción de diversos elementos, es la mejor. 
Si la nodriza tiene las mamas o los pezones demasiados pequeños, será necesario frotar los senos. Se levantarán también las mamas, aplicando de arriba abajo vendaje de apósitos ancho y suelto, y se presionará suavemente, con el fin de que se produzca un aflujo de leche más abundante.

Si a lo largo de la lactancia, la leche se agota, lo mejor es buscar a otra nodriza; si es imposible, se dará leche [a la nodriza], haciendo afusiones abundantes de agua caliente, precedidas de la administración, en forma de bebida, de algún medicamento que pueda traer o dar leche, como, por ejemplo, el hinojo de caballo, que se hará hervir con la dosis de dos cótilas con vino odorífero, o apio caballar administrado de la misma forma, o la raíz de apio.

Después de las afusiones, se hará chupar las mamas por alguien que tirará con violencia; se hará fricciones y nuevas afusiones, y, después del baño, se dará a beber dos cótilas de una cocción de cebollas de puerros, malva, apio, hinojo, o de alguna otra especie de hierba salvaje (decocción que deberá contener trigo muy cocido), se decantará con cuidado y se mezclará, en vez de con aceite con vino odorífero. Se dará una especie de semilla cualquiera cocida con alfalfa de árbol, o con los susodichos ingredientes, y se dará la misma cantidad, después de haber decantado al tamiz el vino. Tras la administración de estos medicamentos, se aplicará una ventosa sobre cada mama, y se ordenará [a alguien] chupar [las mamas] con bastante fuerza; se darán alimentos que tengan poca cohesión y que se distribuyan fácilmente en el cuerpo, y abundantes bebidas, por ejemplo vino odorífero y tenue que se beberá caliente, teniendo cuidado de poner en el agua [que se ha mezclado], alguno de los medicamentos que nosotros acabamos de nombrar. 